\title{
Sclérose en plaques et vaccination contre l'hépatite B : vers la fin d'une appréhension ?
}

Deux articles parus simultanément dans le $\mathrm{New}$ England Journal of M edicine concluent, pour le premier, à l'absence de risque de déclenchement d'une sclérose en plaques ( $S E P$ ) après vaccination contre I'hépatite B [1] et, pour le second, à l'absence de risque de poussée après cette même vaccination [2]. Ces deux articles vont-ils conclure la polémique, essentiellement française, qui a débuté en octobre 1998 alors que les autorités françaises suspendaient la vaccination systématique contre l'hépatite $B$ chez les enfants et adolescents? Depuis cette date, la vaccination contre I'hépatite $B$ a très nettement diminué en France, et les notices des vaccins incluent la SEP dans leurs contre-indications. L'Organisation Mondiale de la Santé avait revu en septembre 1998 les données scientifiques concernant cette vaccination, et n'avait observé aucune preuve définitive de relations avec la SEP. Ainsi, la décision d'arrêter la vaccination systématique chez les enfants et les adolescents avait été motivée par les difficultés pour donner une information complète aux personnes concernées dans le cadre du suivi scolaire. II était cependant recommandé de continuer à vacciner les jeunes enfants et les adultes à risque d'hépatite $B$.

Cette décision avait fait suite à la publication d'études cas-témoins rapportant une augmentation (non significative) du risque de SEP après une vaccination contre l'hépatite $B$ [3], la survenue de cas de SEP chez des sujets jeunes vaccinés, et une base théorique de mimétisme moléculaire entre certains antigènes du virus et des composants de la myéline. La première étude reposait sur une série hospitalière de 121 femmes ayant une
SEP et 121 femmes témoins. Elle montrait un risque relatif de troubles neurologiques dans les 2 mois qui suivent l'exposition au vaccin de 1,7 [0,5$6,3]$. Malgré cette période d'exposition relativement courte, l'absence de certificat de vaccination pour tous les patients exposait à un biais de rappel important. La deuxième étude incluait 152 patients SEP et 153 témoins traités au même moment dans les mêmes hôpitaux pour d'autres maladies neurologiques ne pouvant être liées à un vaccin. Les injections vaccinales étaient évaluées sur la période des 2 mois qui précédait la survenue de la maladie. Le risque relatif de troubles neurologiques au décours du vaccin était de 1,4 [0,4-4,5] dans cette étude. Il est légitime de diminuer la durée de la fenêtre d'exposition si l'on considère que le lien entre la vaccination et l'épisode neurologique n'est possible que dans un intervalle de deux mois. Cependant, l'inconvénient majeur est de réduire la puissance de l'étude. La troisième étude comportait 500 cas de SEP avec 6 témoins par cas. L'exposition aux vaccins était, dans ce cas, considérée pendant les 12 mois précédents et le risque relatif obtenu fut de $1,6[0,6$ à 4]. Le large intervalle de confiance observé s'explique par le fait que cette étude, réalisée au Royaume-U ni, portait sur une population peu exposée à la vaccination contre l'hépatite $B$.

Les résultats de ces travaux ne sont pas confirmés par les deux études récentes. La première est une étude cas-témoins nichée dans des cohortes d'infirmières américaines [1]. Chaque patiente y est appariée à 6 témoins. L'étude a inclus 192 SEP. L'analyse multivariée du risque de SEP après vaccination contre l'hépa- tite $B$, quelle que soit sa date de réalisation, est de $0,9[0,5$ à 1,6$]$, et de 0,7 lorsqu'elle était effectuée dans les 2 ans qui précèdent la maladie. Le risque n'est pas modifié par le nombre de doses ou l'origine du vaccin (recombinant ou non).

La seconde étude évalue le risque de survenue d'une poussée de SEP après vaccination [2]. II s'agit d'une étude de cas-crossover où le patient est son propre témoin. L'exposition aux vaccins au cours d'une période de 2 mois précédant une poussée est comparée à l'exposition pendant plusieurs périodes de 2 mois antérieures à cette période étudiée. Le risque relatif de poussée associée à une vaccination contre l'hépatite $B$ est de $0,67[0,20-2,17]$ chez des patients qui sont indemnes de poussée pendant un an. Des résultats similaires sont observés pour les vaccinations antitétanique, anti-grippale ou en cas d'association de plusieurs vaccins. Ces deux types d'étude ont pour avantage de limiter, dans le premier cas, les biais liés aux erreurs de rappels concernant les vaccins effectivement réalisés, et, dans le second cas, ceux liés au choix des témoins.

Si la réponse ultime atten due, c'est-àdire le risque de développement d'une SEP chez des enfants ou adolescents vaccinés, n'est pas spécifiquement apportée par ces deux études récentes qui portent sur des populations plus âgées [1] ou déjà malades [2], elle avait été donnée, mais de façon moins rigoureuse, dans une étude parue il y a un an et conduisant aux mêmes conclusions [4]. Ces résultats permettront-ils de rassurer définitivement les patients et les médecins sur des observations isolées de possibles «SEP post-vaccinales»? Un 
effet indésirable très précoce et limité dans le temps aurait-il pu expliquer un risque relatif supérieur à 1 (mais non significatif) dans les premières études qui prenaient en compte un délai post-vaccinal de 2 mois et non de 12 [3] ?

La discussion autour de ce vaccin efficace - d'autant plus qu'il est pratiqué à grande échelle - souligne l'intérêt de rechercher l'antigène ou les antigènes cibles de la SEP, les caractéristiques du dérèglement immunologique de cette maladie et les conséquences potentielles d'un phénomène de mimétisme moléculaire entre des agents infectieux et un antigène du système nerveux.

1. Ascherio A, Zhang SM, Hernán MA, et al. Hepatitis $B$ vaccination and the risk of multiple sclerosis. N Engl J M ed 2001; 344: 327-32.

2. Confaureux $C$, Suissa $S$, Saddier $P$, Bourdès $V$, Vukusic S. For The Vaccines In Multiple Sclerosis Study Group. Vaccinations and the risk of relapse in multiple sclerosis. N Engl J M ed 2001; 344: 31926.

3. Touzé E, Gout O, Verdier-Taillefer MH, LyonCaen 0 , Alpérovitch A. Premier épisode de démyélinisation du système nerveux central et vaccination contre l'hépatite $B$ : étude cas-témoins pilote. Rev Neurol (Paris) 2000; 156: 242-6.

4. Sadovnick AD, Scheifele DW. School-based hepatitis $B$ vaccination programme and adolescent multiple sclerosis. Lancet $2000 ; 355: 549-50$

\section{Alain Créange}

Service de neurologie, H ôpital H enriM ondor, 51, avenue du M aréchal-deL attre-de-T assigny, 94010 Créteil Cedex, France et Inserm E.0011, Faculté de médecine de Créteil, France.
Ces merveilleux insectes aux cornes extravagantes. Scarabées, cétoines, hannetons, qui faisaient la joie des enfants de jadis, se font à présent bien rares dans nos campagnes. Avec la diffusion des pesticides a disparu le plaisir d'observer le bousier rouler sa boule, comme le faisait des siècles auparavant le scarabée sacré (Scarabeus sacer) si souvent représenté dans l'Égypte ancienne et symbole du devenir et de la renaissance. Parmi les coléoptères, dans la famille des scarabéidés, le groupe des ontophages comporte à lui seul plus de 2000 espèces à travers le monde. A la différence des hannetons dont les larves ne sont pas coprophages, ces insectes pondent leurs œufs dans des excréments d'animaux et les larves se développent in situ. Pour protéger le développement de leur progéniture, ils déplacent et enterrent, le plus souvent dans des tunnels, ce précieux humus et les mâles se postent à l'entrée pour bloquer le passage et empêcher d'autres mâles d'accéder aux femelles et aux réserves de bouse ou de crottin. Souvent, ils sont pourvus de cornes toutes plus invraisemblables les unes que les autres dont la taille et la position varient énormément d'une espèce à l'autre. On les trouve sur le sommet, ou la base de la tête, ou encore sur le thorax. Elles avaient beaucoup intrigué Darwin qui les avait classées dans les ornements plutôt que dans les attributs de combat [1], ce en quoi il se trompait. Contrairement à certains caractères sexuels $n$ 'apparaissant qu'à l'âge adulte, comme la magnifique queue du paon ou les impressionnants bois des cerfs, les cornes des ontophages sont prévues dans le développement embryonnaire et se forment au détriment des organes voisins: aux dépens des antennes pour celles du sommet de la tête, des yeux pour celles de la base, et des ailes pour celles qui poussent sur le thorax. II s'ensuit, selon les cas une diminution de l'odorat, de la vue ou de la capacité de voler. Un chercheur américain qui avait déjà étudié le mécanisme de développement des cornes des diverses espèces d'ontophages [2], vient de démontrer que la localisation de ces ornements défensifs dépendait du mode de vie et de l'environnement de ces coléoptères [3]. Chez les espèces nocturnes, qui ont besoin d'une bonne vue pour se déplacer la nuit, les cornes ne se développent pas à la base de la tête (aux dépens des yeux). II reste à vérifier que dans un environnement de bouses rares et dispersées, nécessitant de grands déplacements, la localisation sur le thorax (réduisant la taille des ailes) serait évitée, et que la localisation au sommet de la tête (près des antennes) ne s'observerait pas en cas de dispersion rapide des odeurs. S'agit-il d'une nouvelle et spectaculaire démonstration de l'effet d'une pression sélective, chère à Darwin, ou d'un exemple rare de spécification induite par l'environnement, plus proche des théories de Lamarck, la question reste ouverte.

[1. Darwin C. The descent of man and selection in relation to sex, $2^{\text {nd }}$ ed. London: Murray, 1890.]

[2. Emlen D], Nijhout HF. Annu Rev Entomol 2000; 45: 661-9.]

[3. Emlen DL. Science 2001; 291: 1534-6.] 